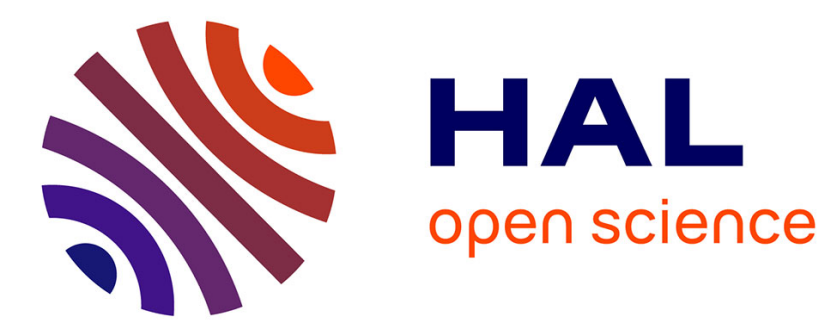

\title{
Performance Comparison of Positioning Techniques in Wi-Fi Networks
}

\author{
Mohamad Yassin, Elias Rachid, Rony Nasrallah
}

\section{To cite this version:}

Mohamad Yassin, Elias Rachid, Rony Nasrallah. Performance Comparison of Positioning Techniques in Wi-Fi Networks. 10th Int. Conf. Innovations in Information Technologies, Nov 2014, Al Ain, United Arab Emirates. 10.1109/INNOVATIONS.2014.6987565 . hal-01310660

\section{HAL Id: hal-01310660 \\ https://hal.inria.fr/hal-01310660}

Submitted on 3 May 2016

HAL is a multi-disciplinary open access archive for the deposit and dissemination of scientific research documents, whether they are published or not. The documents may come from teaching and research institutions in France or abroad, or from public or private research centers.
L'archive ouverte pluridisciplinaire $\mathbf{H A L}$, est destinée au dépôt et à la diffusion de documents scientifiques de niveau recherche, publiés ou non, émanant des établissements d'enseignement et de recherche français ou étrangers, des laboratoires publics ou privés. 


\title{
Performance Comparison of Positioning Techniques in Wi-Fi Networks
}

\author{
Mohamad Yassin*‡, Elias Rachid*, Rony Nasrallah ${ }^{\S}$ \\ *Saint-Joseph University, ESIB, Campus des Sciences et Technologies, Mar Roukoz, Lebanon \\ $\ddagger$ University of Rennes 1, IRISA, Campus de Beaulieu, 35042 Rennes, France \\ $\S$ Alfa Telecommunications, Palm Center, Beirut, Lebanon
}

\begin{abstract}
The wide deployment of Wi-Fi networks empowers the implementation of numerous applications such as Wi-Fi positioning, Location Based Services (LBS), wireless intrusion detection and real-time tracking. Many techniques are used to estimate Wi-Fi client position. Some of them are based on the Time or Angle of Arrival (ToA or AoA), while others use signal power measurements and fingerprinting. All these techniques require the reception of multiple wireless signals to provide enough data for solving the localization problem. In this paper, we describe the major techniques used for positioning in Wi-Fi networks. Real experiments are done to compare the accuracy of methods that use signal power measurement and Received Signal Strength Indication (RSSI) fingerprinting to estimate client position. Moreover, we investigate a fingerprinting method constrained by distance information to improve positioning accuracy. Localization techniques are more accurate when the estimated client positions are closer to the real geographical positions. Accuracy improvements increase user satisfaction, and make the localization services more robust and efficient.
\end{abstract}

Index Terms-Wi-Fi positioning; RSSI fingerprinting; Location Based Services; geolocation

\section{INTRODUCTION}

The recent development of wireless technology, the increasing need for mobile data and the large diversification of wireless devices have led to a wide deployment of Wireless Local Area Networks (WLAN). IEEE 802.11 [1] (commonly known as Wi-Fi) is a widespread Radio Access Technology (RAT) used in wireless networks. It offers mobile users the ability to access the network while they are moving, and to use the various available services via a wireless connection.

The traditional services offered by a Wi-Fi network are: providing wireless internet access for users, allowing interconnectivity between multiple Wi-Fi enabled devices that are present in the same network and connecting distant wired networks via a wireless connection. However, WiFi networks also allow the introduction of numerous Location Based Services [2]. These services are based on the knowledge of mobile user position. They have many innovative applications that are useful for the service providers and for the mobile clients.

Many techniques are used to estimate mobile user geographical position in wireless networks. Time of Arrival (ToA), Time Difference of Arrival (TDoA) [3,4] and Received Signal Strength (RSSI) are techniques that offer range measurements to solve the positioning problem [5]. These measurements are converted into distance information that helps localizing the mobiles.

Positioning in cellular networks $[6,7]$ has the advantage of the previous knowledge of base stations deployment and geographical positions. However, localization accuracy is restrained by the multipath and non-line-of-sight (NLOS) propagation. In Cell-ID [8] localization technique, each mobile estimates its actual position using the geographical coordinates of the serving base station. Other methods make use of the Received Signal Strength (RSS) which is the reception power of the beacon signals transmitted by the base stations $[9,10]$.

Criteria used for localization techniques performance evaluation are: accuracy and cost. They are more accurate when the estimated position is closer to the real geographical position. However, the cost of each technique depends on the number of network nodes (wireless access points) to be involved in the problem, the signaling traffic needed and the processing load of the localization algorithm. Hence, the performance of a localization technique is a compromise between its accuracy and its cost. Other performance metrics [7] are: reliability, latency and applicability.

Localization techniques in WLAN have many applications. These applications are known as Location Based Services (LBS), and they can be classified [2] as emergency services (i.e., security alerts, public safety), informational services (such as news, sports, stocks, etc.), tracking services (like asset/fleet/logistic monitoring or person tracking) and entertainment services (for example: locating a friend and gaming). Specific applications include [11] environment monitoring, support to people with disabilities, consumer protection, meteorology, science, timing, etc.

In this paper, we describe the main techniques used for locating mobile clients in wireless networks. Then we choose techniques based on signal power measurement and Received Signal Strength Indication fingerprinting used for positioning in Wi-Fi networks. In order to compare the performance of these positioning techniques, real experiments are done. We track the movements of a Wi-Fi client in a geographical area covered by three access points of the IEEE 802.11 technology. Localization techniques are more accurate when the estimated client positions are closer to the real geographical positions. Hence, localization accuracy is the main parameter used for performance comparison. An accurate positioning technique in WiFi networks increases user satisfaction, and makes the localization services more robust and efficient.

The rest of the paper is organized as follows: we describe in section (II) the different positioning techniques used in wireless networks. We specify the Wi-Fi localization techniques to be compared in this paper in section (III). Section (IV) contains details about the experiments 
we have made. Experiments results and positioning techniques performance comparison are reported in section $(\mathrm{V})$, and we conclude in section (VI).

\section{RELATED WORK}

Positioning techniques exist in satellite-based networks (e.g. Global Positioning System, GPS), in mobile networks (such as Global System for Mobile communications, GSM) and in Wireless Local Area Networks (like Wi-Fi and Bluetooth).

GPS satellites continuously broadcast their own position, speed and direction. Therefore, each GPS receiver can calculate its own position via trilateration [12]. Receivers position is the intersection of the spheres determined by the distances between the mobile device and each satellite. Localization accuracy depends on the number of visible satellites. Hence, satellite-based positioning does not work well for indoor localization, and even outdoor when facing obstacles such as high buildings.

Assisted-GPS (A-GPS) is introduced to overcome limitations of GPS. Each Base Transceiver Station (BTS) of the mobile network is equipped with GPS receivers. AGPS exploits information about BTS geographical position as well as GPS localization information. However, it requires the upgrade of mobile stations and base transceiver stations.

Cell Identification (Cell-ID) [8] is one of the positioning techniques used in mobile networks where each base station broadcasts both the Location Area Identifier and the Cell-ID to the clients. Having the Cell-ID, each mobile can approximate its position via the geographical coordinates of the corresponding base station.

Triangulation [13] is a positioning technique that exploits the geometry of triangles to locate the mobile clients. It uses the Angle of Arrival (AoA) [14] measurements of several radio links to estimate the communication angle between the base station and the mobile.

In the context of mobile networks, ToA is a time-based technique that evaluates the differences between bursts sent by a mobile. These measurements are converted into distance, and client position is estimated using trilateration. However, ToA requires synchronization between mobiles and base stations. It also depends on the propagation environment which is the main source of error [15].

The position of a mobile is also estimated via trilateration in the Enhanced Observed Time Difference (EOTD) localization technique [16]. The mobile compares the relative times of arrival of bursts transmitted by nearby base stations to calculate its distance from each visible station. E-OTD requires synchronization between mobiles and base stations.

In the next section, we classify and describe the different localization techniques used in Wi-Fi networks.

\section{WI-FI POSITIONING TECHNIQUES}

\section{A. Classification of Wi-Fi positioning techniques}

We classify positioning techniques used in Wi-Fi networks into four main categories: cell identification (CellID), time, RSSI and angle. Methods based on cell identification make use of the radio beacons transmitted by access points. Each user scans the received radio beacons to find out the closer access point from which it receives the greater signal strength.

ToA and TDoA measure the arrival times of signals transmitted by the access point [17]. However, timebased positioning techniques require time synchronization between access points and clients.

The most common positioning techniques in $\mathrm{Wi}-\mathrm{Fi}$ networks are based on the received signal power measurements. Power measurements are converted into quantified levels called Received Signal Strength Indication that reflects the real signal power values. In this context, positioning can use either propagation models or empirical models. Propagation models [18] translate signal strength degradation when moving away from the access point. The signal loss is therefore converted into distance, and client position is estimated via trilateration. However, the empirical model creates a database containing RSSI values for the different points in the positioning map. Client position is then estimated as the point in the map having the closest RSSI values to those measured by the client.

We can measure the angle of arrival of signals transmitted by Wi-Fi clients using directional antennas or antenna arrays. This method is called Angle of Arrival, and it belongs to the category of angle-based positioning techniques.

Fig. 1 summarizes the main categories of Wi-Fi positioning techniques. They are based on Cell-ID, time, RSSI or angle.

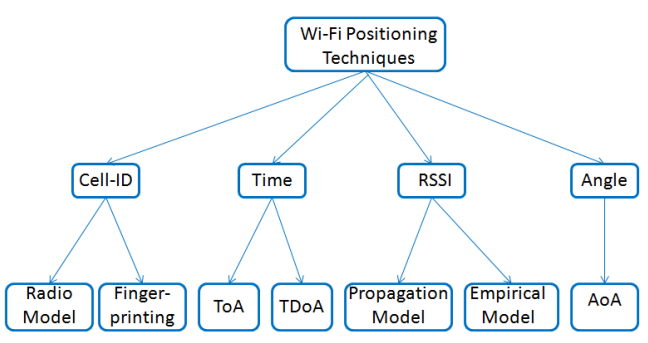

Fig. 1. Classification of Wi-Fi positioning techniques

In this paper, we choose the Received Signal Strength Indication as the main parameter for estimating $\mathrm{Wi}-\mathrm{Fi}$ client position. The remainder of this section contains a description of the RSSI-based positioning techniques to be compared via real tracking experiments.

\section{B. RSSI-based trilateration}

RSSI is converted into distance using propagation models. In fact, radio frequency signal is subject to degradation when propagating in free space. Propagation models formulate signal degradation as a function of the traveled distance and the transmission frequency. The HataOkumura model [19] for instance is used for Path Loss $P L$ prediction in wireless environments. According to this propagation model, $P L$ is given by:

$$
P L=A+B \cdot \log (d)
$$

Given that $d$ is the distance between the access point and the wireless client, $B$ is a factor that depends on antenna characteristics, and $A$ is the fixed loss depending on the transmit frequency. 
The received power $P_{r}$ is a function of the transmit power, antenna gains and path loss. It is given by:

$$
P_{r}=P_{t}+G_{t}+G_{r}-P L
$$

Given that $P_{t}$ is the transmitted power, $G_{t}$ is the transmit antenna gain, and $G_{r}$ is the receive antenna gain.

RSSI gives information about the received power $P_{r}$. Using (1) and (2), we can find the distance $d$ that separates the access point and the Wi-Fi client. Trilateration technique requires three distance measurements to find out client position. Hence, three access points are required to be present in the localization area. Client position is estimated as the intersection of three circles having the access points as centers and the obtained distances as radii.

\section{RSSI fingerprinting}

Received Signal Strength Indication fingerprinting is a positioning technique that uses empirical models. The idea is to perform preliminary RSSI measurements instead of using propagation models. It consists of two phases: an offline phase that generates a radio map for the positioning area, and an online phase that estimates client geographical position.

In the first phase, positioning area is separated into smaller parts representing a grid. We measure RSSI values for each point in the grid. Fig. 2 shows a Wi-Fi localization area where the map is divided into smaller areas.

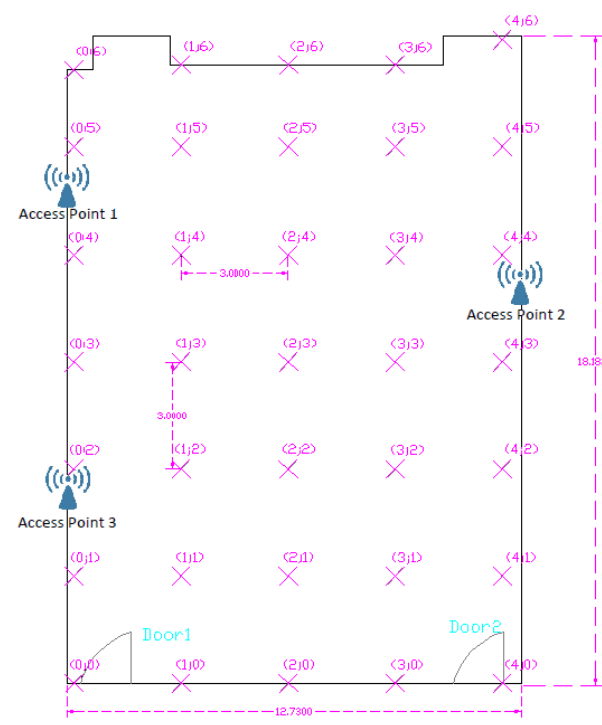

Fig. 2. Localization area divided using a grid

At each point of the grid, Wi-Fi signals are received from the nearby access points. We measure RSSI values under different conditions: time, interference, number of clients, network load. If $n$ is the number of access points in the localization area, an $n$-tuple $\left(\overline{R S S I_{1}}, \overline{R S S I_{2}}, \ldots, \overline{R S S I_{n}}\right)$ containing mean RSSI values is created for each point $(x, y)$ in the map. This information is stored in a database that will be used in the positioning process.

After database creation, we proceed to the position estimation phase. Each Wi-Fi client performs live RSSI measurements. The obtained values are the main parameter when estimating client geographical position. They are

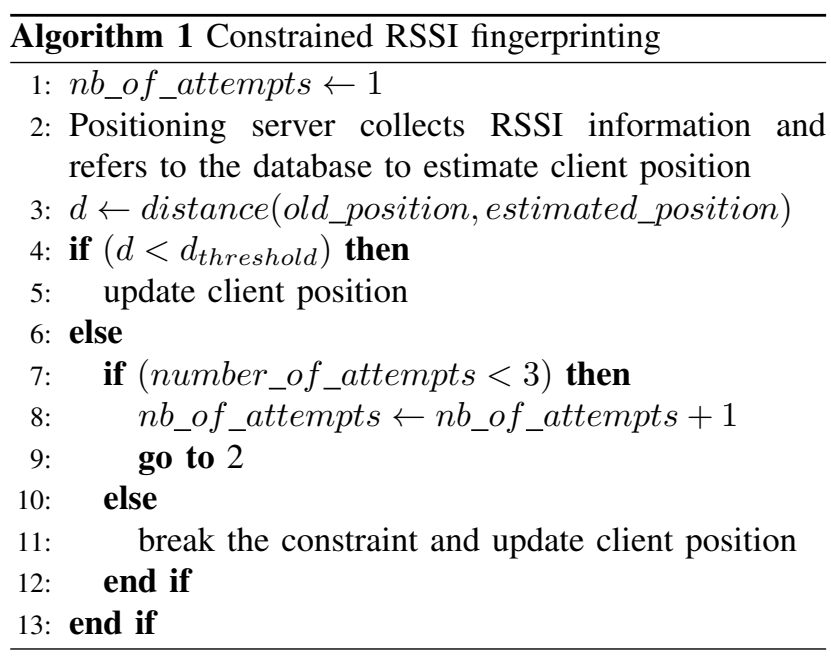

compared to the fingerprint stored in the database to find the closest match. The final user position estimate is the entry in the database that best matches the actual measurements. We use the Euclidean distance to find the stored RSSI $n$-tuple that best matches the current measurements.

\section{Constrained RSSI fingerprinting}

RSSI fingerprinting is used for Wi-Fi client localization. However, its accuracy is restricted by signal fluctuations and by the dynamic characteristics the propagation environment. In order to improve positioning accuracy, we integrate a distance constraint within the position estimation algorithm. In fact, positioning results offer useful information for future position estimation in real-time tracking scenarios.

In this paper, we propose a constrained RSSI fingerprinting method for positioning in Wi-Fi networks. It calculates the distance between the estimated position and the old client position before making positioning decision. The idea is to eliminate position estimations if the distance between the last position and the estimated one is higher than the distance traveled by the Wi-Fi client. Client speed measurement is beyond the scope of this paper; however, we define $d_{\text {threshold }}$ as the maximum distance traveled during the time that separates two consecutive position estimations.

Algorithm 1 describes the constrained RSSI fingerprinting method. The estimated position is approved if it succeeds the distance check. For instance, the distance between the new and the old positions should be less than a predefined distance threshold for $\mathrm{Wi}-\mathrm{Fi}$ clients moving at pedestrian speeds. This threshold depends on client characteristics (i.e., client speed) and on localization area geometry. However, we break the distance constraint after three negative successive checks, and we approve the estimated position for the current client. This algorithm allows us to eliminate positioning errors caused by wireless signal fluctuations. Experiments are performed to verify the performance of the proposed algorithm.

\section{EXPERIMENTS}

A $12.73 \times 18.18 \mathrm{~m}^{2}$ room at the faculty of engineering of Saint-Joseph University in Beirut is chosen as the indoor 
environment for our positioning tests. Our objective is to compare the performance of $\mathrm{Wi}-\mathrm{Fi}$ positioning techniques described in this paper: RSSI-based trilateration, fingerprinting and constrained fingerprinting.

We deploy a Wi-Fi network consisting of three wireless access points as shown in Fig. 2. A Wi-Fi client present in the localization area gets information about the signals transmitted by the three access points deployed in the room.

The localization map is divided into smaller parts via a grid. The distance between two adjacent points in the grid is $3.00 \mathrm{~m}$. We perform RSSI measurements for each point in the map under different conditions: number of clients, network load, etc. Mean RSSI values are stored in the localization database that contains a triplet $\left(\overline{R S S I_{1}}, \overline{R S S I_{2}}, \overline{R S S I_{3}}\right)$ for each point in the grid. Database construction is the first phase of Wi-Fi positioning using fingerprinting technique. In the second phase, live RSSI measurements are compared to the values stored in the database using the Euclidean distance comparison criterion. User position is estimated as the entry in the database that best matches the actual measurements (i.e., that presents the minimum Euclidean distance). However, trilateration converts RSSI values into distance using the propagation models. For a better accuracy, we adapted the propagation models to our propagation environment by performing preliminary signal measurements. Thus, the fixed loss parameter is adjusted according to measurement results.

The centralized approach is used for Wi-Fi client localization. For this reason, a positioning server is deployed in the network. It is connected to the three access points, and it collects all the necessary information about $\mathrm{Wi}-\mathrm{Fi}$ clients present in the system. The server has also access to the localization database. We developed a graphical user interface that shows instantaneous user position on the localization map. Experiment scenarios and results are given in the next section.

\section{EXPERIMENTS RESULTS AND DISCUSSION}

We use three positioning techniques based on RSSI to estimate the position of a pedestrian Wi-Fi client that moves across the room (real-time tracking). Performance comparison parameters are: percentage of erroneous estimations and positioning accuracy.

\section{A. RSSI-based trilateration}

The Wi-Fi client moves along a predefined path that includes 16 points of the map. At each point, position estimation is done via the trilateration method. A centralized server collects the three RSSI measurements required to estimate client position. Fig. 3 shows the real path of the Wi-Fi client and the estimated path using RSSI-based trilateration method.

Trilateration positioning results do not exactly match with the real positions of the Wi-Fi client. In fact, distance calculation is based on instantaneous RSSI measurements. Thus, errors are due to RSSI fluctuations along with time, interference and network load. However, the majority of the estimated positions are close to the real position of the client. The distance between the estimated position and the

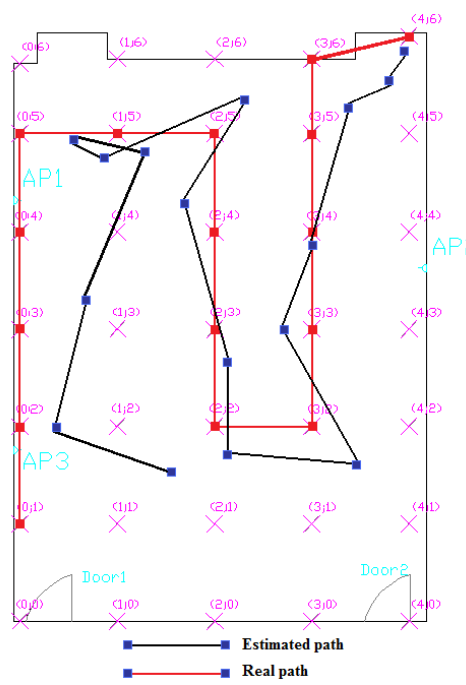

Fig. 3. Real-time tracking using RSSI-based trilateration

real position is less than $3 m$ for approximately $87 \%$ of the positioning decisions. It is the minimum distance between two adjacent points in the grid also called positioning precision.

\section{B. RSSI fingerprinting}

In the second experiment, we use RSSI fingerprinting to estimate user position. The Wi-Fi client moves along the same path under the same network conditions (i.e., number of clients and network load). Fig. 4 shows Wi-Fi client tracking results using RSSI fingerprinting method.

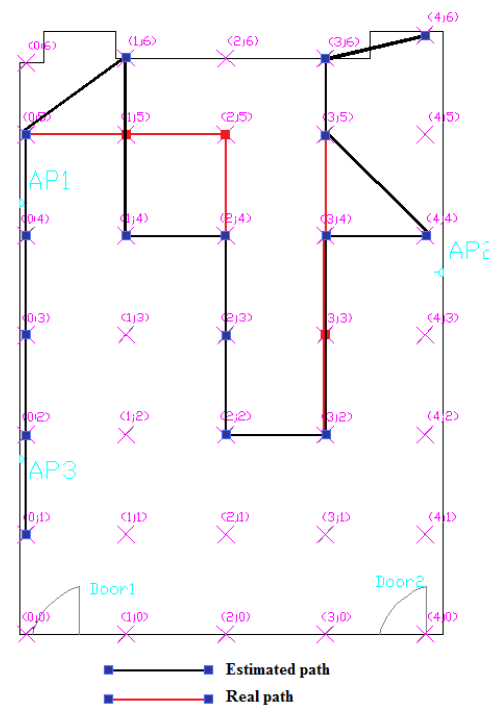

Fig. 4. Wi-Fi client tracking using RSSI fingerprinting

$75 \%$ of the estimated positions using the fingerprinting method exactly match the real positions of the Wi-Fi client. Moreover, erroneous estimations are located in the neighboring of the real client positions. In fact, localization error is comparable to the minimum distance between two adjacent points in the grid $(3 \mathrm{~m})$. In comparison with RSSI-based trilateration, the fingerprinting method shows greater immunity to RSSI fluctuations. Instead of converting instantaneous RSSI measurements into distance, the fingerprinting method compares the measured values with 
the mean RSSI values stored in the database. Positioning decision is made after finding the best match with mean RSSI values. Hence, the impact of RSSI fluctuations is mitigated. Localization results are more robust against interference and network conditions.

\section{Constrained RSSI fingerpriniting}

Positioning decisions are made according to the constrained RSSI fingerprinting method in the third experiment. The Wi-Fi client moves along the same path. Positioning results are shown in Fig. 5. We assume that the client cannot travel more than $3 m$ at pedestrian speed between two consecutive position estimations. Note that the calculation of the distance threshold is beyond the scope of this paper.

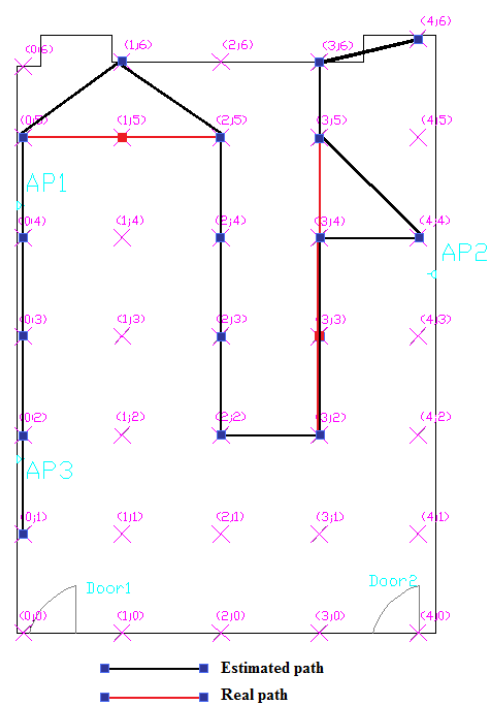

Fig. 5. Wi-Fi positioning using constrained RSSI fingerprinting

Around $81 \%$ of the estimated positions using constrained RSSI fingerprinting exactly match the real client positions. In addition, the distance between erroneous position estimations and real client positions is comparable to the minimum distance between two adjacent points in the grid. Constrained RSSI fingerprinting is also immune to RSSI fluctuations. Experiments results show that positioning accuracy is higher when using the constrained RSSI fingerprinting method. Moreover, the trilateration technique has never found the exact position of the WiFi client. Nevertheless, the main advantage of trilateration is that it does not require a pre-positioning phase where we should perform RSSI measurements to be stored in a database.

\section{CONCLUSION}

In this paper, we described the major techniques used for positioning in wireless networks. They are mainly based on the Received Signal Strength Indication. RSSI fingerprinting methods are more accurate than trilateration; however, trilateration does not require the construction of a fingerprinting database. Accuracy improvements increase user satisfaction, and make the localization services more robust and efficient.

Future work may study localization techniques cost and performance when increasing the number of access points. We can also benefit from the diversity of wireless access networks deployed in the same geographical area to improve the accuracy of positioning techniques. For example, data from mobile networks (such as Global System for Mobile Communications) can be combined with information from Wireless Local Area Networks (such as $\mathrm{Wi}-\mathrm{Fi}$ ) in order to get a more accurate position estimation.

\section{ACKNOWLEDGMENT}

This work was jointly supported by the National Council for Scientific Research (CNRS) Lebanon and Saint Joseph University of Beirut.

\section{REFERENCES}

[1] S. G. Matthew, 802.11 Wireless Networks: The Definitive Guide, O'Reilly, 2002.

[2] D. Mohapatra and S. B. Suma, "Survey of Location Based Wireless Services," in IEEE Int. Conf. Personal Wireless Communications, 2005.

[3] L. Cong and W. Zhuang, "Hybrid TDoA/AoA Mobile User Location for Wideband CDMA Cellular Systems," in IEEE Trans. Wireless Communication, 2002.

[4] D. H. Shin and T. K. Sung, "Comparisons of Error Characteristics Between ToA and TDoA Positioning," in IEEE Trans. Aerospace and Electronic Systems, 2002.

[5] X. Ji and H. Zha, "Robust Sensor Localization Algorithm in Wireless Ad-Hoc Sensor Networks," in Proc. $12^{\text {th }}$ Int. Conf. Computer Communications and Networks, 2003.

[6] T. Kos, M. Grgic, and G. Sisul, "Mobile User Positioning in GSM/UMTS Cellular Networks," in $48^{t h}$ Int. Symp. Multimedia Signal Processing and Communications, Zadar, 2006.

[7] I. K. Adusei, K. Kyamakya, and K. Jobmann, "Mobile Positioning Technologies in CellularNetworks: an Evaluation of their Performance Metrics," in Proc.Military Communications Conf., 2002.

[8] E. Trevisani and A. Vitaletti, "Cell-ID Location Technique Limits and Benefits: an Experimental Study," in Proc. $6^{\text {th }}$ IEEE Workshop Mobile Computing Systems and Applications, 2004.

[9] N. Deblauwe and L. V. Biesen, "Cellular Positioning: from Received Signal Strength Measurements to the Algorithm," in IEEE Global Communications Conf., New Orleans, 2008.

[10] C. M. Huang, T. H. Hsieh, and S. Y. Lin, "A SignalAware Fingerprinting-Based Positioning Technique in Cellular Networks," in $14^{\text {th }}$ Int. Conf. Network-Based Information Systems, Tirana, 2011.

[11] J. J. Peng, "A Survey of Location Based Service for Galileo System," in Int. Symp. Computer Science and Computational Technology, Shanghai, 2008.

[12] B. T. Fang, "Trilateration and Extension to Global Positioning System Navigation," in Journal Guidance, Control and Dynamics, 1986, pp. 715-717.

[13] L. Peneda, A. Azenha, and A. Carvalho, "Trilateration for Indoors Positioning Within the Framework of Wireless Communications," in The $35^{\text {th }}$ Annu. Conf. IEEE Industrial Electronics, Porto, 2009.

[14] A. Pages-Zamora, J. Vidal, and D. H. Brooks, "Closed-Form Solution for Positioning Based on Angle of Arrival Measurements," in The $13^{\text {th }}$ IEEE Int. Symp. Personal, Indoor and Mobile Radio Communications, 2002

[15] P. Deng and P. Z. Fan, "An AoA Assisted ToA Positioning System," in Int. Conf. Communication Technology Proc, Beijing, 2000.

[16] M. Ciurana, S. Cugno, and F. Barcelo-Arroyo, "WLAN Indoor Positioning Based on ToA with Two Reference Points," in $4^{\text {th }}$ Workshop Positioning, Navigation and Communication, Hannover, 2007.

[17] P. Chai and L. Zhang, "Indoor Radio Propagation Models and Wireless Network Planning," in IEEE Int. Conf. Computer Science and Automation Engineering, Zhangiiajie, 2012.

[18] M. Kumar, V. Kumar, and S. Malik, "Performance and Analysis of Propagation Models for Predicting RSS for Efficient Handoff," in Int. Journal Advanced Scientific and Technical Research, 2012.

[19] M. Hassan-Ali and K. Pahlavan, "A New Statistical Model for SiteSpecific Indoor Radio Propagation Prediction Based on Geometric Optics and Geometric Probability," in IEEE Trans. Wireless Communications, 2002. 